\title{
How does semi-automated computer-derived CT measure of breast density compare with subjective assessments to assess mean glandular breast density, in patients with breast cancer?
}

\author{
G J BANSAL, MRCP, FRCR and S KOTUGODELLA, MRCP \\ The Breast Centre, University Hospital of Llandough, Penarth, UK \\ Address correspondence to: Dr Gaurav Jyoti Bansal \\ E-mail: jyoti.bansal@wales.nhs.uk
}

Objectives: (a) To compare radiologists' breast mammographic density readings with $\mathrm{CT}$ subjective measures. (b) To correlate computer-derived measurement of CT density with subjective assessments. (c) To evaluate density distributions in this cohort of patients with breast cancer. Methods: A retrospective review of mammograms and CT scans in 77 patients with breast cancer obtained within 1 year of each other was performed. Two radiologists independently reviewed both $\mathrm{CT}$ and mammograms and classified each case into four categories as defined by the breast imaging-reporting and data system of the American College of Radiology. Inter-reader agreements were obtained for both mammographic and CT density subjective evaluations by using the Cohen-weighted kappa statistic and Spearman correlation. The semi-automated computer-derived measurement of breast density was correlated with visual measurements.
Results: Inter-reader agreements were lower for subjective CT density grades than those for mammographic readings 0.428 [confidence interval $(\mathrm{Cl}), 0.24-0.89$ ] vs $0.571(\mathrm{Cl}$, 0.35-0.76). There was moderately good correlation between subjective CT density grades and the mammographic density grades for both readers $(0.760$ for Reader 1 and 0.913 for Reader 2). The semi-automated CT density measurement correlated well with the subjective assessments, with complete agreement of the density grades in $84.9 \%$ of patients and only one level difference in the rest. Conclusions: Semi-automated CT density measurements in the evaluation of breast density correlated well with subjective mammographic density measurement.

Advances in knowledge: There is good correlation between CT and mammographic density, but further studies are needed on how to incorporate semi-automated CT breast density measurement in the risk stratification of patients.
High glandular density on mammograms has been identified as a risk factor for development of breast cancer. ${ }^{1-3}$ Moreover, mammograms of breasts with high glandular density have low sensitivity for diagnosing breast cancer. There has been an impetus to use methods other than traditional mammograms to evaluate breasts with high density such as ultrasound and MRI. ${ }^{4,5}$

On the other hand, CT scans are increasingly performed in a variety of age groups for reasons other than breast symptoms. Following recent recommendations of low-dose chest CT screening for lung cancer in high-risk patients, ${ }^{6}$ many females have had CT of the chest performed but not mammograms. We believe that CT provides a valuable resource to get reliable information with respect to breast density. If breast density can be determined accurately and is deemed to be high on CT scan, there is a case to suggest supplemental screening with other modalities in addition to mammography.

The aim of this study is to evaluate (1) if semi-automated CT measurement of density is a reliable method to measure breast density by comparing computer-generated values with visual mammographic density values and subjective CT measurements and (2) to evaluate the density distribution in this cohort of breast cancers.

\section{METHODS AND MATERIALS}

This was a retrospective analysis of 109 patients who were treated for breast cancer over a 3-year period (2009-12) and had both mammograms (Hologic ${ }^{\circledR}$ Dimensions $^{\text {TM }}$ Digital, Bedford, MA) and CT scan of the chest within 1 year of each other. All patients had contrast CT of the chest, abdomen and pelvis as per the departmental protocol for 
staging of patients with locally advanced breast cancer. A 64-slice GE CT scanner (CT OPTIMA 660; GE Healthcare, Fairfield, CT) with $0.625-\mathrm{mm}$ slice thickness sections was used. Approximately, $1-1.5 \mathrm{ml} \mathrm{kg}^{-1}$ of contrast was injected, and all the scans were evaluated in the arterial phase of the study at $25 \mathrm{~s}$ after administration of contrast. All scans used an average of $120 \mathrm{kVp}$ and $80 \mathrm{mAs}$, capped at $560 \mathrm{mAs}$. Informed consent or ethical approval was not required, as this was conducted purely as a retrospective analysis. Females who had bilateral mastectomy or had implants or whose breasts were not fully included in the field of view were excluded from the analysis. Similarly, some females had imaging from other centres and therefore either CT scan or mammograms were not available for review for the purpose of this study. Following exclusions, 77 females were appropriate for inclusion in this study. All had CT scan and mammograms within 1 year of each other at our centre. The mean age of our patients was 60.3 years (range, 23-91 years; median, 60 years).

\section{Image analysis}

Two radiologists (JB with 5 years' experience of reading CT and mammograms and SK with 1-year experience) reviewed the CT images independently of each other. Mediastinal window setting (width, $400 \mathrm{HU}$; level, $40 \mathrm{HU}$ ) was used throughout the reading session. When classifying breast density subjectively on CT, the readers were free to use the whole stack of images, including multiplanar reformats. Each radiologist classified the breast density onto one of the four grades as defined by the American College of Radiology breast imaging-reporting and data system (BI-RADS) categories: ${ }^{7}$ grade 1 corresponded to fatty breast with a breast parenchymal density of $0-25 \%$; grade 2 corresponded to scattered fibroglandular tissue with breast parenchymal density of 26-50\%; grade 3 corresponded to heterogeneously dense breast with breast parenchymal density of 51-75\%; and grade 4 were extremely dense breasts with breast parenchymal density of $76-100 \%$. Mammograms were reviewed independently after a minimum of

Figure 1. (a) Right mediolateral oblique (RMLO) view, showing cancer within the lower half. (b) Left mediolateral oblique (LMLO) view. (c) Right craniocaudal view (RCC; cancer just behind the nipple). (d) Left craniocaudal (LCC) view.

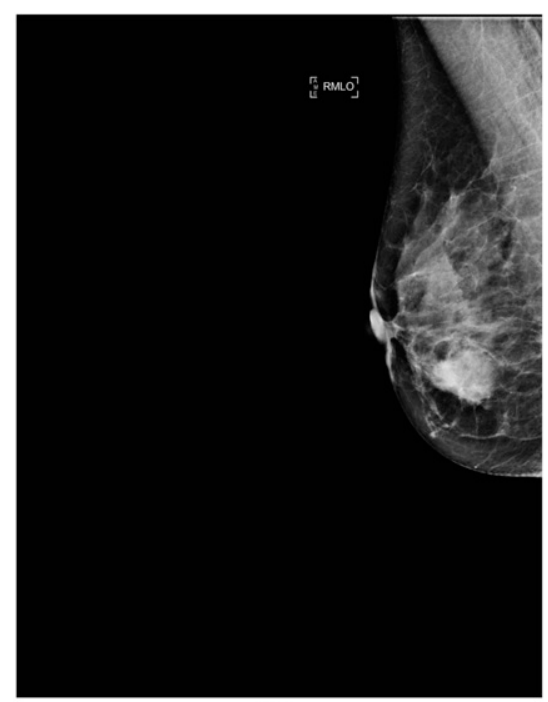

(a)

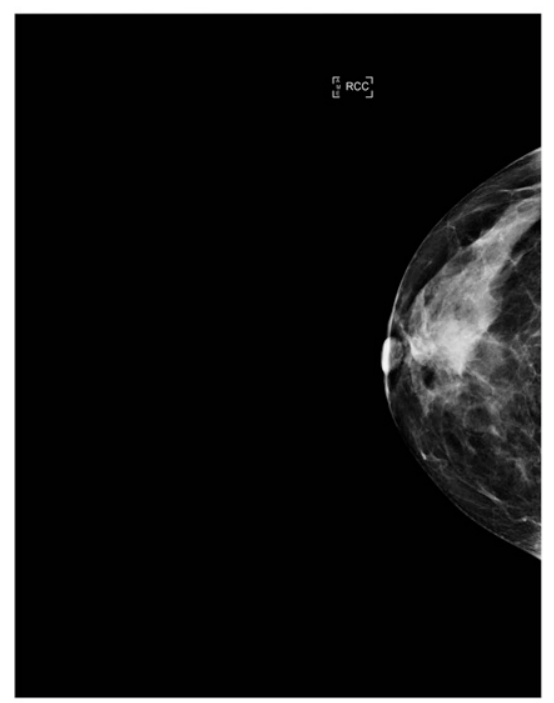

(c)

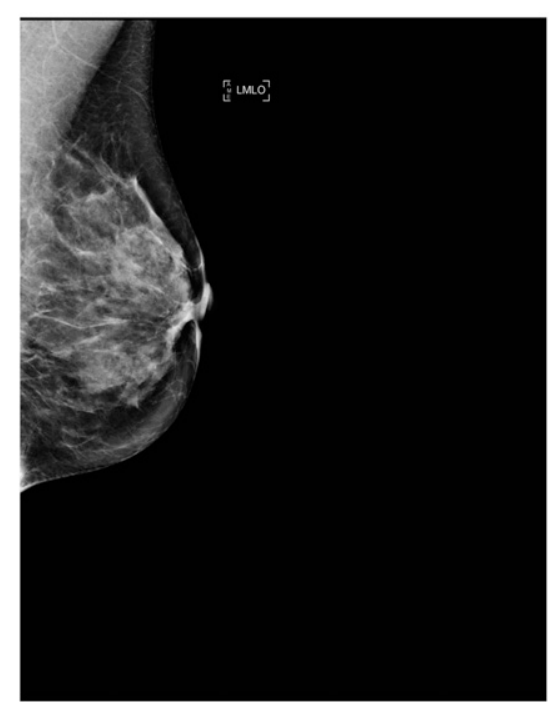

(b)

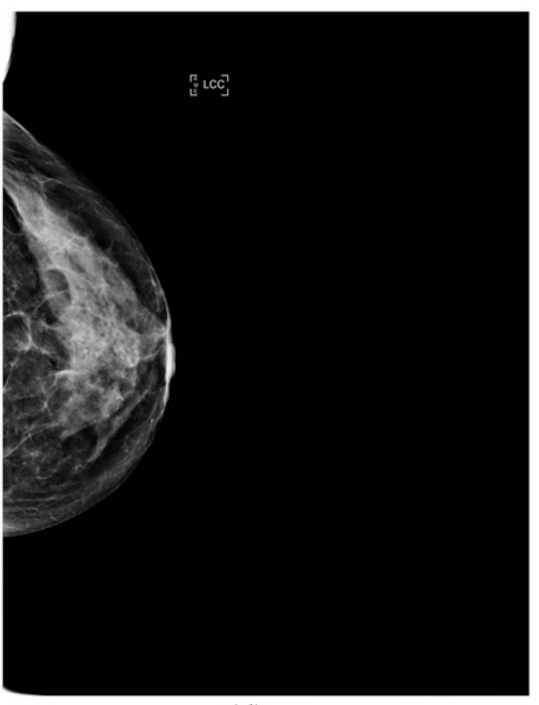

(d) 
1 week in random manner, blinded to the results of CT scan. Both mediolateral oblique and craniocaudal images were used for mammographic density estimation. For both mammograms and CT scan readings, the breast contralateral to known cancer was used for density analysis.

Semi-automated CT analysis of the breast CT density was performed using GE licensed software v. 4.6 on an Advantage Workstation (GE Healthcare). A region of interest was manually outlined at the level of the nipple, which was then partitioned automatically into fat and glandular tissue on the basis of Hounsfield units using histogram threshold technique (Figures $1-3)$. The software then calculated the percentage of glandular tissue in the breast region of interest, assuming a twocompartment model. The computer-derived breast density value was then classified into one of the four BI-RADS density grades and compared with the readings of the radiologists. Manual outlining of the region of interest on CT was performed by one of the readers (SK), blinded to the results of subjective readings. There was a minimum of 1 -week interval between the two measurements (subjective and semi-automated) to reduce memory bias. Moreover, the semi-automated analysis was performed in a random manner, with the sequence of patients different to the subjective readings.

\section{Statistical analysis}

Inter-reader agreements were obtained for both mammographic and CT density evaluations by using the Cohen-weighted kappa statistic. Correlation was also sought between CT density measurement and mammographic density measurements for each reader by using Spearman correlation coefficient, with values ranging from zero (no agreement) to one (full agreement). The semi-automated computer-derived measurement of breast density was correlated with visual measurements. Interpretation of kappa values was performed according to a classification system as follows: $k$ of $0.2-0.4$, fair agreement; $k$ of $0.41-0.60$,

Figure 2. CT scan of the patient shown in Figure 1 with region of interest marked with outline.

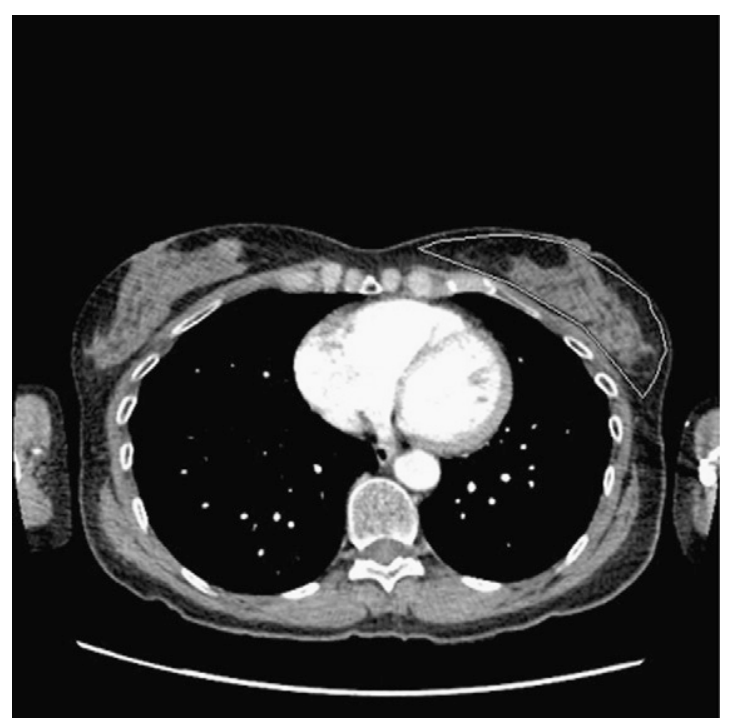

moderate agreement; $k$ of $0.61-0.80$, substantial agreement; $k$ of 0.81-1.00, excellent agreement. SPSS ${ }^{\circledR}$ v. 20 (IBM Inc., Chicago, IL) was used for data analysis, and a $p$-value $<0.05$ was taken as statistically significant.

\section{RESULTS}

Inter-reader agreement was lower for the visual CT density grades than that for mammographic readings, with the kappa value being 0.428 [confidence interval (CI), 0.24-0.89] for CT and 0.571 (CI, 0.35-0.76) for mammographic readings, respectively, although both modalities were in the same moderate agreement category (Tables 1 and 2).

There was substantial correlation between subjective CT density grades and the mammographic density grade for both readers using Spearman correlation coefficient [Reader 1, 0.760 (95\% CI, 0.70-0.85); Reader 2, 0.913 (95\% CI, 0.80-0.95)]. For each reader, there was more disagreement between CT and mammographic readings for lower density grades on both CT and mammograms (grades 1 and 2) than for higher density grades (grades 3 and 4). The semi-automated CT density measurement correlated well with the subjective mammographic assessments, with complete agreement of the density grades in $84.4 \%$ of patients and only one level difference in the remainder for Reader 2. There was $68.8 \%$ agreement between semi-automated CT readings and mammographic readings for Reader 1 and one level difference in the remainder $31.2 \%(p<0.0001)$ (Tables 3 and 4$)$.

This was a cohort of patients with locally advanced breast cancer, with a minimum of 2-3 axillary lymph nodes involved. The most predominant grade of cancer was $2(68 \%)$ followed by $23 \%$ grade 3 . The average size of cancers was $20.5 \mathrm{~mm}$. $49.3 \%$ of patients had CT semi-automated density grade 2 in this cohort of breast cancers, followed by $23.3 \%$ grade 3 , with only $9 \%$ being grade 4 . Therefore, in our study group, about $30 \%$ of patients with breast cancer had breast density $>50 \%$ (grades 3 and 4 ). There was negative correlation between age and automated CT density measurements, with the Pearson correlation coefficient being $r=-0.243$, although this was not statistically significant $(p=0.085)$.

\section{DISCUSSION}

There has been recent debate regarding the value of "one size fits all” mammography screening. Proponents for personalized screening propose individualized screening based on risk assessments and patient preferences. One of the clinical methods used for risk assessments utilizes the Gail model. ${ }^{8}$ The risk factors incorporated in this model include age at menarche, age at first birth, number of first-degree relatives with cancer, number of previous breast biopsies, presence of atypical hyperplasia and breast density.

Mammographic density measurement is therefore valuable to assess risks and offer supplemental screening in patients with dense breasts, which has resulted in increased cancer detection.

There is substantial interobserver variation in reporting mammographic breast density with interobserver kappa values mostly in the moderate category. ${ }^{9}$ In this study, we demonstrated that 
Figure 3. Computer software calculating the glandular breast density.

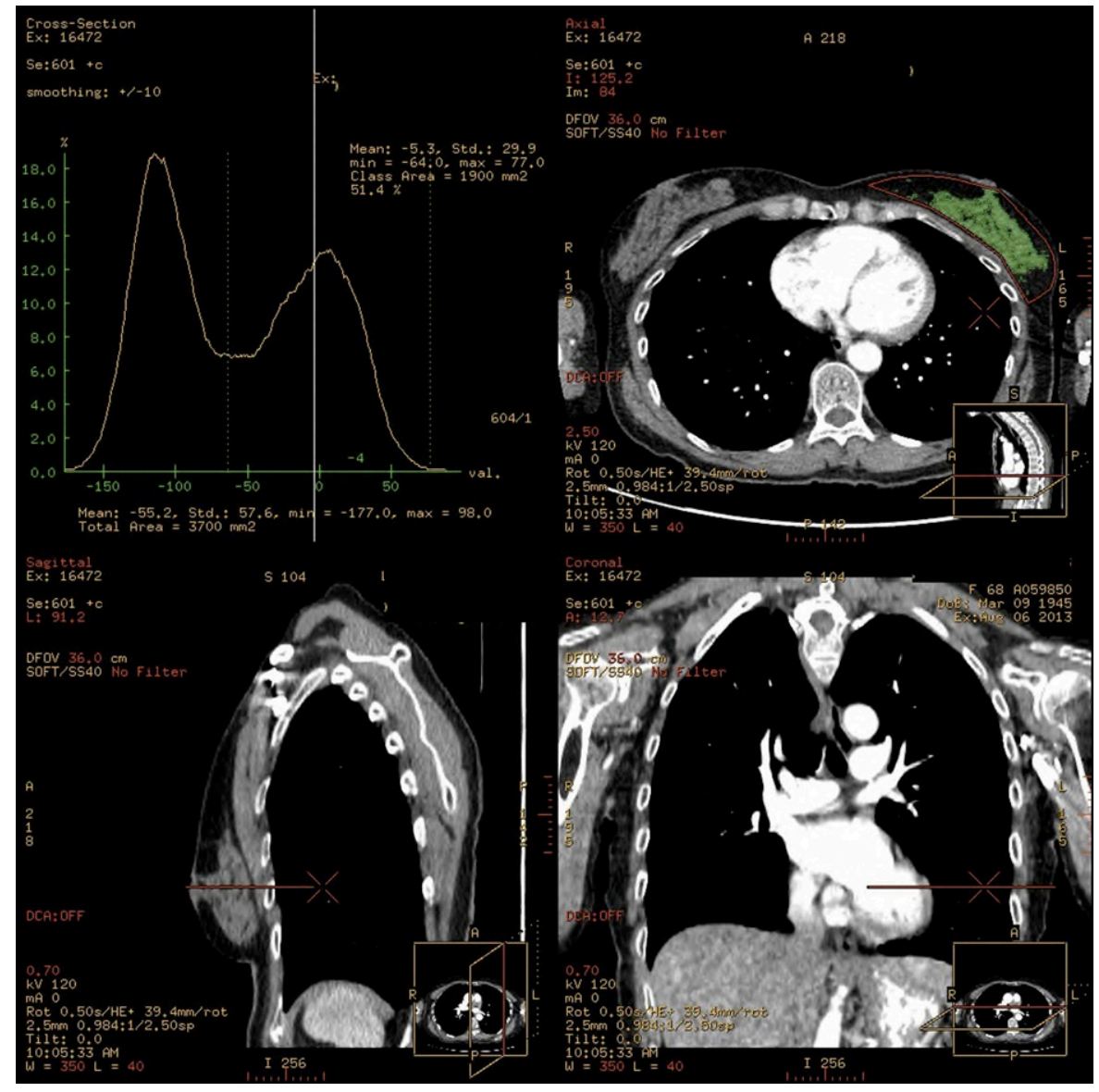

inter-reader agreement of subjective CT readings was no better than that of mammographic readings, contrary to a previous study by Salvatore et al. ${ }^{10}$ Both rely on subjective assessments of breast density percentage based on a visual analysis of a threedimensional structure in two-dimensional plane. Subjective CT readings are further limited by variable positions of the breasts, and there is subjectivity of reviewers in analysing the slice with the maximum breast tissue. These limitations are partly removed with semi-automatic CT measurements, as the region of interest and computer measurements are performed at the nipple level, with the computer generating an actual percentage density.

In our study, there was substantial correlation between CT density grades and the mammographic density grades for both readers. Both Readers 1 and 2 would qualify as inexperienced in terms of
mammographic/CT density readings. This is because there is no obligation to record density readings in either symptomatic or screening setting in routine clinical practice within the UK. The semi-automated CT density measurement correlated well with the subjective mammographic density readings with complete agreement of the readings in $68.0 \%$ for Reader 1 and $84.4 \%$ for Reader 2, and only one level difference in the remainder. We believe the semi-automated technique will be useful for inexperienced readers owing to removal of subjectivity. Other studies ${ }^{10-12}$ corroborate our findings. Moon et $\mathrm{al}^{11}$ compared CT and mammograms of 69 asymptomatic Asian females and suggested that breast density measured from chest CT images correlated well with mammography and were reproducible. The authors used a fuzzy c-mean clustering CT-based quantification method. In a post-mortem study by Johnson et al, ${ }^{12}$ using cone

Table 1. Inter-reader agreement between readers for breast density on CT

\begin{tabular}{|l|c|c|c|c|}
\hline \multirow{2}{*}{ Reader 1 } & \multicolumn{3}{|c|}{ Reader 2 } \\
\cline { 2 - 5 } & Grade 1 & Grade 2 & Grade 3 \\
\hline Grade 1 & $9(11.0 \%)$ & $7(9.1 \%)$ & $1(1.3 \%)$ \\
\hline Grade 2 & $5(6.5 \%)$ & $25(32.5 \%)$ & $5(6.5 \%)$ \\
\hline Grade 3 & & $6(7.8 \%)$ & $8(10.4 \%)$ \\
\hline Grade 4 & & & $1(1.3 \%)$ \\
\hline
\end{tabular}


Table 2. Inter-reader agreement between readers for breast density on mammography

\begin{tabular}{|l|c|c|c|c|}
\hline \multirow{2}{*}{ Reader 1 } & \multicolumn{4}{|c|}{ Reader 2 } \\
\cline { 2 - 5 } & Grade 1 & Grade 2 & Grade 3 & Grade 4 \\
\hline Grade 1 & $8(10.4 \%)$ & $9(11.7 \%)$ & $4(5.2 \%)$ & \\
\hline Grade 2 & $3(3.9 \%)$ & $28(36.4 \%)$ & $9(11.7 \%)$ & $3(3.9 \%)$ \\
\hline Grade 3 & & $4(5.2 \%)$ & $5(6.5 \%)$ & $4(5.2 \%)$ \\
\hline Grade 4 & & & \\
\hline
\end{tabular}

beam CT (CBCT) with fuzzy c-mean algorithm and involving 40 post-mortem breasts, the authors found automatic CT measures of breast density more efficient and reduced interobserver variation. Salvatore et $\mathrm{al}^{10}$ further evaluated a subset of 40 patients with semi-automated CT density measurement and found agreement in $90 \%$ of cases. In our study, we found our semi-automated CT density measurements agreed with subjective mammographic assessments in up to $84.4 \%$ of cases, with only one level difference in the rest. Similar results to ours were found in other studies involving three-dimensional imaging techniques such as tomosynthesis ${ }^{13}$ and dedicated breast $\mathrm{CT}^{14}$ which had better inter-reader variation than twodimensional techniques.

In our study group, about $30 \%$ of patients with breast cancer had breast density $>50 \%$ (grades 3 and 4 ). Although high breast density is associated with increased risk of cancer, most breast cancers occur in females with none of the commonly associated risk factors. The population in a study by Evans et $\mathrm{al}^{15}$ comprised asymptomatic screening population. Their population had $15.25 \%$ females with breast density $>50 \%$. Our cohort of breast cancer patients had about $30 \%$ patients with breast density of 3 or more.

There are a few limitations of our study. First, this is a small pilot study involving only 77 CT-mammogram pairs. Further studies with more numbers would help to validate our findings. Second, we had two radiologists with limited-to-moderate experience of reading mammographic and CT density. This may account for only moderate agreement in subjective readings. However, we feel semi-automated CT measurements are a useful way to assess density, especially for less experienced radiologists. Thirdly, our technique of semi-automatic CT density measurement is not fully automatic and still relies on reader marking an appropriate region of interest. However, we feel this method is more objective than subjective eyeballing. Another limiting factor is the lack of a true gold standard. A true gold standard for breast density evaluations is lacking other than histopathological evaluation of mastectomy specimens. Fully automated software for evaluation of breast density have limited availability on only GE or Hologic digital mammography systems. Previously published studies assessing breast density with fully automated methods also lack this true gold standard. Asymmetrical distribution of glandular tissue in the breast may further confound the results. This was a semi-automated analysis, and all attempts were made to standardize the measurement of glandular tissue, through measurement at the nipple level. It was presumed that maximum glandular tissue lay behind the nipple level and ptotic breasts could be accounted for as both chest and abdomen were scanned in this staging CT.

Within the context of UK National Health Service Breast Screening Programme (NHSBSP), a recent ambitious population-based study concluded that mammographic density and genetic factors should be considered in addition to standard risk factors for determining breast cancer risk. This was perceived to require more frequent screening. ${ }^{15}$ The authors concluded that automated measurements of breast mammographic density would be easier to adapt to the now universal digital screening programmes. We believe, if the same information could be reliably derived from CT performed for other reasons, this information can be fed back to females to assess their risk of developing cancer. There is also a US legislation mandating that females should be informed of their breast density reported on mammograms. ${ }^{4,5}$

The extra information that can be derived from CT ordered for purposes other than the key indication is not new. ${ }^{16} \mathrm{CT}$ has been used for coronary artery calcification measurements ${ }^{17}$ and bone density measurements. ${ }^{18}$ We believe, semi-automated breast density measurements will prove to be a valuable resource for risk assessments of patients.

Table 3. Reader 1: correlation of semi-automated breast density and mammography

\begin{tabular}{|l|c|c|c|c|}
\hline \multirow{2}{*}{ CT-automated values } & \multicolumn{4}{|c|}{ Mammographic readings (Reader 1) } \\
\cline { 2 - 5 } & Grade 1 & Grade 2 & Grade 3 & Grade 4 \\
\hline Grade 1 & $10(13.0 \%)$ & $4(5.2 \%)$ & 0 & 0 \\
\hline Grade 2 & $3(3.9 \%)$ & $26(33.8 \%)$ & $9(11.7 \%)$ & 0 \\
\hline Grade 3 & 0 & $6(7.8 \%)$ & $2(15.6 \%)$ & $5(6.5 \%)$ \\
\hline Grade 4 & 0 & 0 & $2.6 \%)$ & 0 \\
\hline
\end{tabular}


Table 4. Reader 2: correlation of semi-automated breast density and mammography

\begin{tabular}{|l|c|c|c|c|}
\hline \multirow{2}{*}{ CT-automated values } & \multicolumn{4}{|c|}{ Mammographic readings (Reader 2) } \\
\cline { 2 - 5 } & Grade 1 & Grade 2 & Grade 3 & Grade 4 \\
\hline Grade 1 & $12(15.6 \%)$ & $2(2.6 \%)$ & 0 & 0 \\
\hline Grade 2 & $4(5.2 \%)$ & $32(41.6 \%)$ & $2(2.6 \%)$ & 0 \\
\hline Grade 3 & 0 & $4(5.2 \%)$ & $14(18.2 \%)$ & 0 \\
\hline Grade 4 & 0 & 0 & 0 & $7(9.1 \%)$ \\
\hline
\end{tabular}

In summary, our study demonstrated that whilst subjective CT breast density measurement was no better than mammographic density measurements, both lying within the moderate agreement category range, semi-automated CT breast density assessment is a reliable method to assess breast density by removing subjectivity with good correlation with mammographic density.

\section{REFERENCES}

1. Boyd NF, Martin LJ, Rommens JM, Paterson AD, Minkin S, Yafee MJ, et al. Mammographic density: a heritable risk factor for breast cancer. Methods Mol Biol 2009; 472: 343-60. doi: 10.1007/978-160327-492-0_15

2. Razzaghi H, Troester MA, Gierach GL, Olashn AF, Yankaskas BC, Millikan RC. Mammographic density and breast cancer risk in White and African American women. Breast Cancer Res Treat 2012; 135: 571-80. doi: 10.1007/s10549-012-2185-3

3. Tamimi RM, Byrne C, Colditz GA, Hankinson SE. Endogenous hormones levels, mammographic density, and subsequent risk of breast cancer in postmenopausal women. J Natl Cancer Inst 2007; 99: 1178-87.

4. Weigert J, Steenbergen S. The connecticut experience: the role of ultrasound in the screening of women with dense breasts. Breast J 2012; 18: 517-22. doi: 10.1111/ tbj. 12003

5. Hooley RJ, Greenberg KI, Stackhouse RM, Geisel JL, Butler RS, Philpotts LE. Screening US in patients with mammographically dense breasts: initial experience with Connecticut Public Act 09-41. Radiology 2012; 265: 59-69.

6. Kovalchik SA, Tammemagi M, Berg CD, Caporaso NE, Riley TL, Korch M, et al. Targeting of low dose CT screening according to the risk of lung-cancer death. $N$ Engl J Med
2013; 369: 245-54. doi: 10.1056/ NEJMoa1301851

7. American College of Radiology. American College of Radiology breast imaging reporting and data system (BI-RADS). 4th edn. Reston VA: American College of Radiology; 2003.

8. Gail MH, Brinton LA, Byar DP, Corle DK, Green SB, Schairer C, et al. Projecting individualized probabilities of developing breast cancer for white females who are being examined annually. J Natl Cancer Inst 1989; 81: 1879-86.

9. Berg WA, Campassi C, Langenberg P, Sexton MJ. Breast imaging reporting and data system: inter- and intraobserver variability in feature analysis and final assessment. AJR Am J Roentgenol 2000; 174: 1769-77.

10. Salvatore M, Margolies L, Kale M, Wisnivesky J, Kotkin S, Henschke CI, et al. Breast density: comparison of chest CT with mammography. Radiology 2014; 270: 67-73. doi: 10.1148/radiol.13130733

11. Moon WK, Lo CM, Goo JM, Bae MS, Chang JM, Huang CS, et al. Quantitative analysis for breast density estimation in low dose chest CT scans. J Med Syst 2014; 38: 21. doi: 10.1007/s10916-014-0021-5

12. Johnson T, Ding H, Le HQ, Ducote JL, Molloi S. Breast density quantification with cone-beam CT: a post mortem study. Phys Med Biol 2013; 58: 8573-91.
13. Kontos D, Bakic PR, Carton AK, Troxel AB, Conant EF, Maidment AD. Parenchymal texture analysis in digital breast tomosynthesis for breast cancer risk estimation: a preliminary study. Acad Radiol 2009; 16: 283-98. doi: 10.1016/j.acra.2008.08.014

14. Nelson TR, Cervino LI, Boone JM, Lindfors KK. Classification of breast computed tomography data. Med Phys 2008; 35: 1078-86.

15. Evans DG, Warwick J, Astley SM, Stavrinos P, Sahin S, Ingham S, et al. Assessing individual breast cancer risk within the UK national health service breast screening programme: a new paradigm for cancer prevention. Cancer Prev Res (Phila) 2012; 5: 943-51. doi: 10.1158/1940-6207.CAPR-11-0458

16. Lee CI, Forman HP. What we can and cannot see coming. Radiology 2010; 257: 313-14. doi: 10.1148/radiol.10101437

17. Shemesh J, Henschke CI, Shaham D, Yip R, Farooqi AO, Cham MD, et al. Ordinal scoring of coronary artery calcification on low-dose CT scans of the chest is predictive of death from cardiovascular disease. Radiology 2010; 257: 541-8. doi: 10.1148/ radiol. 10100383

18. Budoff MJ, Hamirani YS, Gao YL, Ismaeel H, Flores FR, Child J, et al. Measurement of thoracic bone mineral density with quantitative CT. Radiology 2010; 257: 434-40. doi: 10.1148/radiol.10100132 